\title{
Bundespräsident Horst Köhler - eine (kritische) Analyse
}

von Gerd Strohmeier und Ruth Wittlinger

\begin{abstract}
Die Rolle des Bundespräsidenten wird als vor allem zeremonieller, repräsentativer und integrativer Art beschrieben. Allerdings scheint sich diese Rolle unter Horst Köhler bedeutend gewandelt zu haben. Bei einer oberflächlichen Betrachtung - zumindest der ersten Amtsperiode von Horst Köhler - stellt sich der Eindruck ein, dass dieser von seinen Befugnissen deutlich mehr Gebrauch gemacht hat als sein(e) Vorgänger. Dies wird im Rahmen des vorliegenden Beitrags durch eine ausführliche Analyse der Rechtsgrundlagen des Amtes des Bundespräsidenten und dessen Ausübung durch Horst Köhler untersucht. Die Autoren kommen dabei zu dem Ergebnis, dass das Amt des Bundespräsidenten seit Köhlers Wahl 2004 politisiert wurde, dies aber mehr auf Köhlers Kommentare zur Tagespolitik zurückzuführen ist als auf die Ausnutzung seiner formalen Kompetenzen.
\end{abstract}

The role of the Federal President is usually described as being of a ceremonial, representative and integrative nature. This appears to have changed significantly under Horst Köhler - at least in his first term in office. On the surface, President Köhler seems to have made significantly more use of his powers during this period than any of his predecessors. The article critically examines the powers of the Federal President and the way Horst Köhler has exercised them. The authors conclude that the German Presidency has indeed been politicised since Köhler's election in 2004 although this has been due to his comments on day-to-day politics rather than his use of the given political powers.

\section{Einleitung}

Aufgrund der katastrophalen Erfahrungen mit dem Reichspräsidenten der Weimarer Republik wurden die politischen Kompetenzen des Bundespräsidenten deutlich reduziert. Seine Rolle wird deshalb als vor allem zeremonieller, repräsentativer und integrativer Art beschrieben. Infolgedessen ist sie der des britischen Monarchen ähnlich und kann mit Walter Bagehot als ,dignified part of the constitution" "1 gesehen werden. Allerdings scheint sich diese Rolle unter Horst Köhler bedeutend gewandelt zu haben. Bei einer oberflächlichen Betrachtung zumindest der ersten Amtsperiode - stellt sich der Eindruck ein, dass Köhler von

1 Bagehot, W.: The English Constitution, London, 1964, 61. 
seinen Befugnissen deutlich mehr Gebrauch gemacht hat als sein(e) Vorgänger. Dies soll im Rahmen des vorliegenden Beitrags durch eine ausführliche Analyse der Rechtsgrundlagen des Amtes des Bundespräsidenten und dessen Ausübung durch Köhler untersucht werden. Dabei werden zunächst die in der Verfassung festgelegten und in der Verfassungspraxis langfristig herausgebildeten Funktionen bzw. Kompetenzen des Bundespräsidenten dargestellt. Daran schließt eine Untersuchung der Amtsführung durch Köhler an. Dabei werden u.a. seine Entscheidung zur Auflösung des Bundestages im Juli 2005, seine wiederholte Weigerung, Gesetze der Großen Koalition auszufertigen, und seine Entscheidung, frühere RAF-Terroristen nicht zu begnadigen, untersucht. Abschließend wird festgestellt, ob und inwiefern unter Köhler eine verstärkte Politisierung des Bundespräsidentenamtes stattgefunden hat.

\section{Funktionen und Kompetenzen des Bundespräsidenten und ihre bisherige Ausübung durch frühere Bundespräsidenten}

Der Bundespräsident hat eine Reihe von Funktionen bzw. Kompetenzen, welche (direkt oder indirekt) im Grundgesetz verankert sind, jedoch nicht immer angemessen durch die Verfassung präzisiert werden. Der Parlamentarische Rat war vorrangig bestrebt, die politische Macht des Staatsoberhauptes zu beschränken. Dabei versäumte er es jedoch, die Kompetenzen des Bundespräsidenten klar zu umreißen: „Die Abkehr vom Amt eines machtvollen Staatsoberhauptes hat so sehr die Debatten über die Stellung des Bundespräsidenten geprägt, dass schließlich nur noch recht unklar herausgearbeitet wurde, was eigentlich positiv die Aufgabe des Bundespräsidenten als Staatsoberhaupt sei“. ${ }^{2}$ In der Folge mussten die bestehenden Unklarheiten konkretisiert werden: durch die (herrschende) Staatsrechtslehre sowie durch die Staatspraxis.

Der Bundespräsident nimmt grundsätzlich all die symbolischen Funktionen wahr, welche üblicherweise mit dem Amt des Staatsoberhauptes verbunden sind. Diese größtenteils zeremoniellen und repräsentativen Funktionen verleihen dem Bundespräsidenten naturgemäß keine wesentlichen politischen Befugnisse. Gleichwohl ist der Bundespräsident dadurch in der Lage, politisch Einfluss zu nehmen. Letztlich obliegen ihm ,indirekte, nicht an formale Kompetenzen gebundene Einflussmöglichkeiten auf das politische Leben, die nicht unterschätzt

2 Patzelt, W. J.: Der Bundespräsident, in: Gabriel, O. u.a. (Hg.): Handbuch Politisches System der Bundesrepublik Deutschland, München, 2005, 291-308, hier 295. 
werden dürfen“"3, und die durch „,[e]ine Verbindung von Charme und quasisakraler Ausstrahlung wie sie Richard v. Weizsäcker gegeben war" ${ }^{\text {“4 }}$ durchaus potenziert werden können. Diese „,informalen Kompetenzen“ des Bundespräsidenten resultieren im Endeffekt ,aus der selbstverständlichen Autorität seines Amtes, die diesem vom GG offensichtlich zugedacht und die ihm unter den bedeutenden Gestalten, die es seit 1949 ausgeübt haben, auch im Bewusstsein des Staatsvolks zugewachsen ist“沾. Dazu zählt nicht zuletzt die Möglichkeit, durch politische Reden Einfluss zu nehmen, wovon die Bundespräsidenten in der Geschichte der Bundesrepublik relativ häufig Gebrauch gemacht haben. Dabei äußerten sie sich in der bisherigen Staatspraxis zum Teil relativ „,unverblümt“ zu (tages-)politischen Themen - und gelegentlich auch gegen die amtierende Bundesregierung. Zu denken ist hier an die Äußerungen von Richard v. Weizsäcker zur Frage des Regierungssitzes, ${ }^{6}$ die Forderung von Roman Herzog nach einem „Ruck durch Deutschland“" oder die Kritik an der Parteipolitik des Bundesrats durch Johannes $\mathrm{Rau}^{8}$. Derart deutliche - und vor allem regierungskritische Worte von Bundespräsidenten sind jedoch nicht allzu häufig bzw. nur in Sonderfällen zu beobachten gewesen und stellen in der Geschichte der Bundesrepublik eher die Ausnahme als die Regel dar. Davon abgesehen hat der Bundespräsident jedoch auch erhebliche politische Kompetenzen, die sich bekanntlich in zwei Gruppen aufteilen lassen: Prüfungskompetenzen and Gestaltungskompetenzen.

\section{Prüfungskompetenzen}

Prüfungskompetenzen können als rechtliche oder notarielle Kompetenzen verstanden werden, welche den Bundespräsidenten mit einer begrenzten, aber keineswegs unbedeutenden politischen Befugnis versehen: Sie ermächtigen ihn zur (verfassungs-)rechtlichen Kontrolle anderer Staatsorgane. Die Prüfungskompetenzen des Bundespräsidenten sind nicht direkt im Grundgesetz verankert, kön-

3 Sontheimer, K. /Bleek, W.: Grundzüge des politischen Systems der Bundesrepublik Deutschland, München, 1997, 329.

4 Patzelt, W. J., a.a.O., 306.

5 Herzog, R.: Art. 58 GG, in: Maunz T./Dürig F.: Grundgesetz Kommentar, München, Mai 1986, Lfg. 25 , Rdnr. 17.

6 Vgl. v. Beyme, K.: Das politische System der Bundesrepublik Deutschland, München/Zürich, 1996, 290.

7 Vgl. Herzog, R.: Aufbruch ins 21. Jahrhundert, Ansprache von Bundespräsident Roman Herzog im Hotel Adlon am 26.04.1997. Diese und alle nachfolgend zitierten Reden der Bundespräsidenten sind unter http://www.bundespraesident.de abrufbar und werden von dort zitiert.

8 Vgl. Rau, J.: Erklärung zur Ausfertigung des Zuwanderungsgesetzes am 20.06.2002 im Schloss Bellevue in Berlin. 
nen aber aus Art. 20 Abs. 3 GG abgeleitet werden: „Die Gesetzgebung ist an die verfassungsmäßige Ordnung, die vollziehende Gewalt und die Rechtsprechung sind an Gesetz und Recht gebunden." Infolgedessen ist der Bundespräsident an das (Verfassungs-)Recht gebunden und kann nicht gegen dieses handeln. Im Gegenteil: Der Bundespräsident hat ,das Grundgesetz und die Gesetze des Bundes [zu] wahren und [zu] verteidigen“, wie es auch in seinem Amtseid (Art. 56 GG) heißt.

Die wichtigste Prüfungskompetenz des Bundespräsidenten betrifft die Gesetzesausfertigung, welche in Art. 82 Abs. 1 GG geregelt ist: „Die nach den Vorschriften dieses Grundgesetzes zustande gekommenen Gesetze werden vom Bundespräsidenten nach Gegenzeichnung ausgefertigt und im Bundesgesetzblatte verkündet." Entsprechend der herrschenden Staatsrechtslehre impliziert die Ausfertigung von Gesetzen durch den Bundespräsidenten das Recht (und die Pflicht) des Staatsoberhauptes, sie auf ihre formelle (verfahrensrechtliche) sowie auf ihre materielle (inhaltliche) Verfassungsmäßigkeit zu überprüfen. Ohne Zweifel erhält der Bundespräsident mit dem (umstritteneren) materiellen Gesetzesprüfungsrecht einen größeren politischen Spielraum als mit dem formellen Prüfungsrecht. Grundsätzlich bewegt sich der Bundespräsident durch das Gesetzesprüfungsrecht zwischen dem Parlament, das Gesetze verabschiedet, und dem Bundesverfassungsgericht, dem das Recht der Letztentscheidung über die Verfassungsmäßigkeit von Gesetzen zukommt. Die Diskussion über die Reichweite des Prüfungsrechts, also die Frage, ob der Bundespräsident eine Vollprüfung oder nur eine Evidenzkontrolle vorzunehmen hat, ist weitestgehend theoretisch ausgerichtet. Schließlich haben die Bundespräsidenten bislang äußerst sparsam von ihrem Gesetzesprüfungsrecht Gebrauch gemacht und dabei stets eine Evidenzkontrolle vorgenommen, d.h. Gesetze nur dann nicht unterzeichnet, wenn sie aus ihrer Sicht „Zweifelsfrei und offenkundig“ verfassungswidrig waren. Insgesamt wurden bis zum Amtsantritt von Horst Köhler sechs Gesetze in der Geschichte der Bundesrepublik von den jeweiligen Bundespräsidenten nicht ausgefertigt: Theodor Heuss verweigerte 1951 die Ausfertigung des Gesetzes zur Durchführung des Art. 108 Abs. 2 GG aus formellen Gründen (aufgrund fehlender Zustimmung des Bundesrats). Davor hatte Heuss - was damals noch möglich war - ein Rechtsgutachten des Bundesverfassungsgerichts zu der zwischen Bundesrat und Bundestag umstrittenen Zustimmungsbedürftigkeit des Gesetzes eingeholt, welches dieses bejahte. Heinrich Lübke verweigerte 1960 die Ausfertigung des Gesetzes über den Betriebs- und Belegschaftshandel aus materiellen Gründen (wegen eines Verstoßes gegen die Freiheit der Berufswahl und Be- 
rufsausübung gem. Art. 12 Abs. 1 GG). Davor hatte auch Lübke ein wissenschaftliches Gutachten eingeholt. Gustav Heinemann verweigerte 1969 die Ausfertigung des Gesetzes zur Änderung des Gesetzes zum Schutz der Berufsbezeichnung „Ingenieur“ aus formellen Gründen (wegen fehlender Gesetzgebungskompetenz des Bundes). Davor hatte das Bundesverfassungsgericht das ursprüngliche Ingenieurgesetz ,wegen fehlender Gesetzgebungskompetenz des Bundes für die bloße Regelung der Bedürfnisse zum Führen einer Berufsbezeichnung für nichtig erklärt" ${ }^{\star 9}$. Aus den gleichen Gründen verweigerte Heinemann 1970 die Ausfertigung des Architektengesetzes. Walter Scheel verweigerte 1976 die Ausfertigung des Gesetzes zur Änderung des Wehrpflichtgesetzes und des Zivildienstgesetzes aus formellen Gründen (wegen fehlender Zustimmung des Bundesrats). Davor hatte Scheel die zwischen Bundesrat und Bundestag umstrittene Zustimmungsbedürftigkeit des Gesetzes eingehend geprüft. Später ist die Zustimmungsbedürftigkeit des Bundesrates vom Bundesverfassungsgericht in einem anderen Zusammenhang bestätigt worden. Richard von Weizsäcker verweigerte 1991 die Ausfertigung des zehnten Gesetzes zur Änderung des Luftverkehrsgesetzes aus materiellen Gründen (wegen eines Verstoßes der geplanten privatrechtlichen Organisation der Bundesanstalt für Flugsicherung gegen Art. 33 Abs. 4 und Art. 87d Abs. 1 GG). Schließlich wurde das Gesetz nach einer entsprechenden Grundgesetzänderung erneut verabschiedet und auch ausgefertigt. $^{10}$

Diese wenigen Beispiele machen deutlich, dass die deutschen Bundespräsidenten im Regelfall Gesetze ausfertigten - darunter auch eine Reihe von Gesetzen, die später vom Bundesverfassungsgericht (nach einer entsprechenden Vollprüfung) für verfassungswidrig erklärt wurden. Dabei hätte die Verfassungswidrigkeit einiger dieser Gesetze durchaus auch im Rahmen einer Evidenzkontrolle, also „Zweifelsfrei und offenkundig“, festgestellt werden können. Schließlich haben Bundespräsidenten nicht selten äußerst „,heikle“ Gesetze trotz Zweifeln an deren Verfassungsmäßigkeit ausgefertigt - und auf die bestehende Klagemöglichkeit vor dem Bundesverfassungsgericht hingewiesen. So fertigten Karl Carstens, Roman Herzog und Johannes Rau verfassungsrechtlich keineswegs unbedenkliche Gesetze aus, da sie einen Verfassungsverstoß „zweifelsfrei und offenkundig“

9 Schindler, P.: Datenhandbuch zur Geschichte des Deutschen Bundestages 1949 bis 1999, Bd. 2, BadenBaden, 1999, 2454.

10 Vgl. Schindler, P., a.a.O., 2453ff.; Rau, J.: Vom Gesetzesprüfungsrecht des Bundespräsidenten, in: Deutsches Verwaltungsblatt, 119/1 (2004), 1-8, hier 1ff. 
nicht feststellen konnten. ${ }^{11}$ Johannes Rau hat 2001 sogar das auf spektakuläre Weise zustande gekommene „Zuwanderungsgesetz“ 2001 ausgefertigt, obwohl es eigentlich ganz offensichtlich (,zweifelsfrei und offenkundig“) formell verfassungswidrig war. Dabei zeigt sich, dass die sich dem Bundespräsidenten stellende Frage, ob ein Gesetz „Zweifelsfrei und offenkundig“ verfassungswidrig ist, maßgeblich davon abhängt, was der jeweilige Amtsinhaber unter ,zweifelsfrei und offenkundig“ versteht. Insgesamt wird jedoch deutlich, dass die Bundespräsidenten nur äußerst zurückhaltend und nach sorgfältiger Abwägung bzw. fachkundiger Information von ihrem Prüfungsrecht Gebrauch gemacht haben.

Über weitere Prüfungskompetenzen verfügt der Bundespräsident im Rahmen der völkerrechtlichen Vertretung der Bundesrepublik Deutschland (Art. 59 Abs. 1 GG), der Ernennung und Entlassung der Bundesregierung, d.h. der Ernennung und Entlassung des Bundeskanzlers und der Bundesminister (Art. 63 Abs. 2 GG; Art. 64 Abs. 1 GG), der Ernennung und Entlassung von Bundesrichtern, Bundesbeamten und Bundeswehroffizieren und -unteroffizieren (Art. 60 Abs. 1 GG) und der Genehmigung der Geschäftsordnung der Bundesregierung (Art. 65 GG). Entscheidend ist, dass der Bundespräsident in all diesen Fällen allein die Rechtmäßigkeit des vorzunehmenden Aktes zu prüfen hat und keinerlei politischen Gestaltungs- oder Ermessensspielraum besitzt. Er darf also weder einen Bundeskanzler ,am Bundestag vorbei“ ernennen noch einen vom Bundestag mit der erforderlichen Mehrheit gewählten Bundeskanzler aus politischen Gründen ablehnen. Wichtig ist in diesem Zusammenhang auch, dass in den meisten Fällen von konstruierten bzw. groben Rechtsverletzungen abgesehen - kaum rechtliche Verstöße denkbar sind, die der Bundespräsident im Rahmen seiner Prüfungskompetenz feststellen könnte. Schließlich fehlt es ,nicht am Prüfungsrecht des Bundespräsidenten [...], sondern an den Rechtsnormen, anhand deren dieses Prüfungsrecht realisiert werden könnte“12. In der Folge führten die Prüfungskompetenzen ,in der Praxis nicht sehr weit ${ }^{\text {“13 }}$. Ein Eingreifen des Bundespräsidenten in den o.a. Fällen war letztlich nur durch ein „Hinausgreifen“ über die bestehende Prüfungskompetenz möglich: auf dem Wege der Ersetzung der verfassungsrechtlich vorgesehenen Prüfung durch eine verfassungsrechtlich nicht vorgesehene politische Entscheidung. Dies ist in der Geschichte der Bundesre-

11 Vgl. Rau, J., a.a.O., 5f.

12 Herzog, R.: Art. 64 GG, in: Maunz T./Dürig F.: Grundgesetz Kommentar, München, April 1983, Lfg. 21, Rdnr. 10.

13 Herzog, R., Art. 64 GG, a.a.O., Rdnr. 10. 
publik jedoch nur äußerst selten der Fall gewesen. So verweigerte 1953 Theodor Heuss die erneute Ernennung von Thomas Dehler zum Bundesjustizminister ${ }^{14}$ erfolgreich, da Konrad Adenauer den Ernennungsvorschlag zurücknahm und nicht vor dem Bundesverfassungsgericht klagte. Dies lässt sich wohl weniger mit dem Respekt vor dem Bundespräsidenten, als vielmehr damit erklären, dass Adenauer ,dieses Veto gegen einen schwierigen Mitarbeiter nicht ungelegen kam und die Koalition dadurch nicht gefährdet wurde“. ${ }^{15}$ Ist der Bundeskanzler aber nicht bereit, das politische Eingreifen des Bundespräsidenten zu akzeptieren, hat Letzterer de facto ,genauso viel Macht, wie der Bundeskanzler schlechte Nerven hat". ${ }^{16}$ Aus diesem Grunde war Theodor Heuss mit Blick auf die Verhinderung eines Bundesministers auch ,erst- und letztmals [!] erfolgreich“. ${ }^{17}$. Allerdings konnten sich Bundespräsidenten - ohne Vorliegen eines durch das Prüfungsrecht gedeckten Grundes - erfolgreich gegen die Ernennung bzw. Entlassung staatlicher Hoheitsträger gemäß Art. 60 Abs. 1 GG weigern. Schließlich „haben zumindest die beiden ersten Bundespräsidenten - Heuss und Lübke - in einer Reihe von Fällen Ernennungen aus politisch-moralischen Gründen erfolgreich verweigert; meist ging es dabei um NS-belastete Personen““ ${ }^{18}$ So weigerte sich Lübke 1965, Carl Creifeld (entgegen dem Votum des Richterwahlausschusses) zum Bundesrichter zu ernennen ${ }^{19}$ - erfolgreich, da Creifeld verzichtete. Ein „Hinausgreifen“ über die Prüfungskompetenz ist, abgesehen von diesen Einzelfällen in der Anfangszeit der Bundesrepublik Deutschland jedoch nicht zu registrieren. Dabei ist allerdings erneut hervorzuheben, dass ein derartiges „Hinausgreifen“ (macht-)politisch nur solange möglich ist, als keine Organklage oder gar Präsidentenanklage gegen das Staatsoberhaupt erfolgt. ${ }^{20}$ Somit kann der Bundespräsident ,,in Fällen, in denen zwar nicht seine rechtliche, wohl aber seine mora-

14 Der Grund für die Weigerung war Dehlers offene Kritik am Bundesverfassungsgericht. Vgl. Hübner, E.: Parlament und Regierung in der Bundesrepublik Deutschland, München, 2000, 233.

15 v. Beyme, K., a.a.O., 287.

16 Konrad Adenauer, zit. in: Becker, K.: Wieviel Macht soll der Bundespräsident haben?, in: Die Zeit, 19.11.1976, Nr. 48, 3.

17 Hübner, E., a.a.O., 233.

18 Rudzio, W.: Das politische System der Bundesrepublik Deutschland, Opladen, 1991, 326.

19 Der Grund für die Weigerung war Creifelds NS-Vergangenheit. Vgl. v. Beyme, K., a.a.O., 287.

20 Schließlich stellt - im Falle der Ernennung der Bundesminister - ,die Inaussichtstellung der Weigerung die Inaussichtstellung einer Verfassungsverletzung dar, die dem Bundeskanzler die Möglichkeit einer Verfassungsklage nach Art. 93 I Nr. 1 gibt und die den Bundespräsidenten letztlich sogar der Gefahr einer Präsidentenanklage nach Art. 61 aussetzt“. Herzog, R., Art. 64 GG, a.a.O., Rdnr. 15. 
lische Position stark ist, durchaus einmal seinen Willen durchsetzen“21 - allerdings nur einmal oder einige wenige Male, keineswegs ständig. Grundsätzlich kann festgehalten werden, dass die meisten der o.g. Befugnisse - die Genehmigung der Geschäftsordnung der Bundesregierung, das Abschließen völkerrechtlicher Verträge, die Ernennung und Entlassung der Bundesregierung ebenso wie die der Bundesrichter, Bundesbeamten und Bundeswehroffiziere - de facto „symbolische Befugnisse“ sind.

Ferner ist darauf hinzuweisen, dass alle in diesen Bereichen getroffenen Entscheidungen des Bundespräsidenten grundsätzlich nur vorübergehender Natur sind. Wenn sich der Bundespräsident - aus (falschen) rechtlichen Bedenken oder anderen Gründen - weigert, eine gewisse, ihm durch das Grundgesetz prinzipiell auferlegte Handlung zu vollziehen, kann er durch das Bundesverfassungsgericht dazu verpflichtet werden.

\section{Gestaltungskompetenzen}

Gestaltungskompetenzen sind prinzipiell uneingeschränkte Kompetenzen des Bundespräsidenten, die diesem ein politisches Ermessen einräumen und unter Umständen eine erhebliche politische Wirkung entfalten. Diese Gestaltungskompetenzen sind direkt im Grundgesetz verankert und werden meist in Krisensituationen bedeutsam, in denen der Bundespräsident häufig zu einem potentiellen „Krisenvermittler und Schiedsrichter“ avanciert. ${ }^{22}$

Zwei wesentliche Gestaltungskompetenzen des Bundespräsidenten betreffen die Regierungsbildung. Eine davon bezieht sich auf die in Art. 63 Abs. 1 GG verankerte Wahl des Bundeskanzlers: „Der Bundeskanzler wird auf Vorschlag des Bundespräsidenten vom Bundestage ohne Aussprache gewählt.“ Dem Vorschlagsrecht des Bundespräsidenten kommt allerdings nur bei unklaren Mehrheitssituationen eine signifikante politische Bedeutung zu. In diesem Fall könnte der Bundespräsident in den Regierungsbildungsprozess eingreifen bzw. diesen zu lenken versuchen. Als Folge der Verhältniswahl besteht in Deutschland normalerweise die Notwendigkeit zur Bildung einer Koalitionsregierung. Sind allerdings die Parteien nicht fähig oder gewillt, eine (stabile) Koalitionsregierung zu bilden, kann der Bundespräsident eine entscheidende politische Rolle im Prozess

21 Herzog, R.: Art. 60 GG, in: Maunz T./Dürig F.: Grundgesetz Kommentar, München, Mai 1986, Lfg. 25, Rdnr. 18.

22 Vgl. Schmidt, M. G.: Political Institutions in the Federal Republic of Germany, Oxford, 2003, 37. 
der Regierungsbildung übernehmen. Mit anderen Worten: Wenn eine Partei oder eine Koalition von Parteien über die absolute Mehrheit der Sitze im Bundestag verfügt, kann der Bundespräsident letztlich keinen Einfluss auf die Wahl des Bundeskanzlers ausüben. Er muss dann letztlich den Kandidaten vorschlagen, der erwartungsgemäß die Stimmen der Mehrheit der Bundestagsmitglieder erhält. Gibt es jedoch keine Partei oder Parteienkoalition mit einer absoluten Mehrheit der Sitze im Bundestag, könnte der Bundespräsident den Regierungsbildungsprozess wesentlich beeinflussen. ${ }^{23}$ In der Geschichte der Bundesrepublik ist dies bisher allerdings nie der Fall gewesen. Es gab immer eine Partei oder normalerweise - eine Parteienkoalition mit einer absoluten Mehrheit im Parlament. Allerdings kann die zunehmende Fragmentierung und Ausdifferenzierung des Parteienspektrums, die Herausbildung eines Fünf-Parteien-Systems mit einer fehlenden Mehrheit für eine minimal winning coalition aus einer großen und einer kleinen Partei sowie einer als koalitionsunwürdig eingestuften Partei in Zukunft dazu führen, dass klare Mehrheiten fehlen, die Möglichkeiten zur Regierungsbildung abnehmen und der Gestaltungsspielraum des Bundespräsidenten entsprechend zunimmt.

Wird der vom Bundespräsidenten vorgeschlagene Kandidat nicht gewählt, kann der Bundestag unabhängig vom Bundespräsidenten einen Bundeskanzler vorschlagen und wählen. Dies ist in Art. 63 Abs. 3 GG festgelegt: „Wird der Vorgeschlagene nicht gewählt, so kann der Bundestag binnen vierzehn Tagen nach dem Wahlgange mit mehr als der Hälfte seiner Mitglieder einen Bundeskanzler wählen." Gelingt es jedoch nicht, innerhalb von 14 Tagen einen Bundeskanzler mit der absoluten Mehrheit zu wählen, könnte eine weitere Gestaltungsfunktion des Bundespräsidenten ,aktiviert“ werden, die in Art. 63 Abs. 4 GG geregelt ist: „Kommt eine Wahl innerhalb dieser Frist nicht zustande, so findet unverzüglich ein neuer Wahlgang statt, in dem gewählt ist, wer die meisten Stimmen erhält. Vereinigt der Gewählte die Stimmen der Mehrheit der Mitglieder des Bundestages auf sich, so muss der Bundespräsident ihn binnen sieben Tagen nach der Wahl ernennen. Erreicht der Gewählte diese Mehrheit nicht, so hat der Bundespräsident binnen sieben Tagen entweder ihn zu ernennen oder den Bundestag aufzulösen." Mit anderen Worten: Wenn der Bundestag nicht in der Lage ist, einen Bundeskanzler mit mehr als der Hälfte seiner Mitglieder zu wählen, kann der Bundespräsident entweder einen Minderheitenkanzler ernennen oder den Bundestag auflösen. In der Geschichte der Bundesrepublik Deutschland gab es 
allerdings immer einen Kandidaten, der eine solche absolute Mehrheit auf sich vereinigen konnte. Die sich in jüngster Zeit herausbildenden politischen Konstellationen könnten jedoch dazu führen, dass der Bundestag in Zukunft nicht (mehr) in der Lage ist, einen Mehrheitskanzler zu wählen, und in der Folge der Gestaltungsspielraum des Bundespräsidenten zunimmt.

Zwei weitere bedeutende Gestaltungskompetenzen des Bundespräsidenten betreffen die Folgen einer verlorenen Vertrauensfrage des Bundeskanzlers. Eine dieser Befugnisse bezieht sich auf die für diesen Fall in Art. 68 Abs. 1 GG geregelte Möglichkeit der Auflösung des Bundestages: „Findet ein Antrag des Bundeskanzlers, ihm das Vertrauen auszusprechen, nicht die Zustimmung der Mehrheit der Mitglieder des Bundestages, so kann der Bundespräsident auf Vorschlag des Bundeskanzlers binnen einundzwanzig Tagen den Bundestag auflösen. Das Recht zur Auflösung erlischt, sobald der Bundestag mit der Mehrheit seiner Mitglieder einen anderen Bundeskanzler wählt.“ Demnach ist es dem Bundespräsidenten möglich, den Bundestag auf Vorschlag des Bundeskanzlers nach einer verlorenen Vertrauensfrage aufzulösen, er ist aber nicht dazu verpflichtet.

In der Geschichte der Bundesrepublik wurde die Vertrauensfrage bisher fünfmal gestellt: 1972 (durch Willy Brandt), 1982 (durch Helmut Schmidt), 1982 (durch Helmut Kohl), 2001 (durch Gerhard Schröder) und 2005 (erneut durch Schröder). Drei davon erhielten nicht die erforderliche Unterstützung im Bundestag. In all diesen Fällen wurde der Bundestag durch den Bundespräsidenten auf Vorschlag des Bundeskanzlers aufgelöst. Der Bundespräsident hat folglich immer dem Wunsch des Bundeskanzlers entsprochen - selbst wenn der Kanzler eine sogenannte ,unechte Vertrauensfrage“ stellte. Eine „echte Vertrauensfrage“ liegt dann vor, wenn der Kanzler aufrichtig um die Zusicherung der Unterstützung seiner Politik bittet und sich dieser nicht mehr sicher ist. Dies war etwa 2001 der Fall, als Gerhard Schröder die Vertrauensfrage an die innerhalb seiner Regierungsmehrheit umstrittene Entscheidung über die Entsendung deutscher Truppen nach Afghanistan koppelte. Eine „unechte Vertrauensfrage“ liegt vor, wenn der Kanzler einen Teil seiner Regierungskoalition instruiert, ihm das Vertrauen zu versagen, um vorgezogene Wahlen herbeizuführen. ${ }^{24}$ Zwei Bundeskanzler Helmut Kohl 1982 und Gerhard Schröder 2005 - haben eine solche ,unechte“ Vertrauensfrage gestellt, in der klaren Absicht den Bundestag aufzulösen, obwohl sie über eine relativ stabile Mehrheit im Bundestag verfügten. Das Bundes-

24 Vgl. Heckötter, U./Spielmann, C.: Schröder's Dissolution of the Bundestag Approved, in: European Constitutional Law Review, 2/1 (2006), 5-20, hier 7. 
verfassungsgericht hat beide „unechten Vertrauensfragen“ für verfassungskonform erklärt. Wären sie verfassungswidrig gewesen, hätten die Bundespräsidenten nicht das Recht zur Auflösung des Budestags gehabt. Da sie es jedoch nicht waren, konnten die Bundespräsidenten - wie bei einer ,echten“ Vertrauensfrage - entscheiden, ob sie den Bundestag auflösen oder nicht.

Weigert sich der Bundespräsident, den Bundestag aufzulösen, wird unter Umständen eine andere Gestaltungskompetenz des deutschen Staatsoberhauptes relevant - die Möglichkeit der Erklärung des in Art. 81. Abs. 1 GG geregelten Gesetzgebungsnotstandes: „Wird im Falle des Artikel 68 der Bundestag nicht aufgelöst, so kann der Bundespräsident auf Antrag der Bundesregierung mit Zustimmung des Bundesrates für eine Gesetzesvorlage den Gesetzgebungsnotstand erklären, wenn der Bundestag sie ablehnt, obwohl die Bundesregierung sie als dringlich bezeichnet hat. Das gleiche gilt, wenn eine Gesetzesvorlage abgelehnt worden ist, obwohl der Bundeskanzler mit ihr den Antrag des Artikels 68 verbunden hatte. "Da der Bundestag nach einem negativen Ausgang der Vertrauensfrage bisher immer durch den Bundespräsidenten aufgelöst wurde, ist dieser Fall in der Geschichte der Bundesrepublik Deutschland noch nie eingetreten. Es ist auch in Zukunft bei Vertrauensfragen mit negativem Ausgang grundsätzlich zu erwarten, dass der Bundespräsident dem Vorschlag des Bundeskanzlers (und dem ,indirekten Auflösungsvotum“ des Bundestags) nachkommt und das Parlament auflöst. Die Legitimierung der ,unechten“ Vertrauensfrage durch das Bundesverfassungsgericht und die „präsidiale Auflösungsbereitschaft“ haben dieses Instrument de facto in ein ,indirektes“ Parlamentsauflösungsrecht des Bundeskanzlers überführt. Folglich ist es relativ unwahrscheinlich, dass die Rolle des Bundespräsidenten in diesem Bereich an Bedeutung gewinnen wird.

Eine weitere Gestaltungskompetenz des Bundespräsidenten bildet die in Art. 39 Abs. 3 GG verankerte Möglichkeit, eine Sondersitzung des Bundestages einzuberufen: „Der Bundestag bestimmt den Schluss und den Wiederbeginn seiner Sitzungen. Der Präsident des Bundestages kann ihn früher einberufen. Er ist hierzu verpflichtet, wenn ein Drittel der Mitglieder, der Bundespräsident oder der Bundeskanzler es verlangen." Das bedeutet, dass der Bundespräsident den Bundestagspräsidenten verpflichten kann, eine Sondersitzung des Bundestages einzuberufen, wenn er dies für erforderlich hält. Allerdings kann der Bundespräsident lediglich eine Sondersitzung veranlassen, jedoch keine politische Entscheidung des Bundestages o.ä. herbeiführen. Bisher hat es in der Geschichte der Bundesrepublik noch nie eine Sondersitzung des Bundestages auf Verlangen des Bundespräsidenten gegeben. 
Eine weitere, wenn auch weniger gewichtige Gestaltungskompetenz des Bundespräsidenten besteht darin, den Bundeskanzler oder einen Bundesminister zu verpflichten, die Amtsgeschäfte bis zur Ernennung eines Nachfolgers weiterzuführen. Dies ist in Art. 69 Abs. 3 GG geregelt: „Auf Ersuchen des Bundespräsidenten ist der Bundeskanzler, auf Ersuchen des Bundeskanzlers oder des Bundespräsidenten ein Bundesminister verpflichtet, die Geschäfte bis zur Ernennung seines Nachfolgers weiterzuführen." Infolgedessen kann der Bundespräsident eine „Lücke“ in der Führung der Amtsgeschäfte der Bundesregierung während einer Phase des politischen Wechsels unterbinden, indem er das „Leben“ der alten Bundesregierung verlängert. Bei dieser Befugnis handelt es sich letztlich jedoch um einen eher trivialen Akt, der sich in Übergangszeiten nahezu automatisiert abspielt.

Eine letzte Gestaltungskompetenz des Bundespräsidenten ist das Recht zur Begnadigung: „Er übt im Einzelfalle für den Bund das Begnadigungsrecht aus“ (Art. 60 Abs. 2 GG). Allerdings ist das Recht zur Begnadigung ausschließlich in Einzelfällen sowie bei erstinstanzlichen Strafverfahren vor Gerichten des Bundes möglich und als solches kein politisches Recht im engeren Sinne. Es stellt vielmehr eine juristische Befugnis dar, die jedoch eine bedeutende politische Wirkung entfalten kann - vor allem dann, wenn es sich bei der zu begnadigenden Person um einen politisch motivierten Straftäter handelt. Allerdings ist grundsätzlich festzustellen, dass sich das Begnadigungsrecht des Bundespräsidenten in der Geschichte der Bundesrepublik - aufgrund des „engen Zuschnitts“ und der Delegation des Begnadigungsrechts in den meisten Fällen ${ }^{25}$ - ,durchaus in Grenzen hält‘.26 und relativ wenig politische Beachtung fand.

\section{Die Präsidentschaft von Horst Köhler}

Horst Köhler wurde am 1. Juli 2004 als Nachfolger von Johannes Rau zum neunten Bundespräsidenten der Bundesrepublik Deutschland gewählt. Trotz seiner CDU-Mitgliedschaft besetzte Köhler vor seinem Amtsantritt sowohl im Auftrag der Union als auch der Sozialdemokraten eine Reihe von Schlüsselpositionen. So nahm er Anfang der 1990er Jahre an den Verhandlungen mit der DDR-Führung zur Währungsunion, an Gesprächen mit Moskau über den Abzug

25 Vgl. Stern, K.: Das Staatsrecht der Bundesrepublik Deutschland, Band 2, München, 1980, 264.

26 Herzog, R., Art. 60 GG, a.a.O., Rdnr. 34. 
von Sowjettruppen aus dem Gebiet der DDR sowie an den Verhandlungen über die europäische Wirtschafts- und Währungsunion teil. Auf Wunsch von Helmut Kohl wurde er 1998 Präsident der Europäischen Bank für Wiederaufbau und Entwicklung in London, auf Vorschlag von Bundeskanzler Gerhard Schröder folgte im Mai 2000 die Wahl zum Geschäftsführender Direktor des Internationalen Währungsfonds in Washington (IWF). Schließlich wurde er Anfang Mai 2004 wurde er zum Präsidentschaftskandidaten der CDU/CSU und FDP. Wie erwartet schlug Horst Köhler die SPD-Kandidatin Gesine Schwan aufgrund der klaren Mehrheit der bürgerlichen Parteien in der Bundesversammlung im ersten Wahlgang mit absoluter Mehrheit. Am 1. Juli 2009 wurde Köhler erneut zum Bundespräsidenten gewählt - im ersten Wahlgang mit 613 Stimmen und damit so knapp wie möglich. ${ }^{27}$ Zwar verfügte das schwarz-gelbe Lager keine absolute Mehrheit in der Bundesversammlung, doch fand Horst Köhler auch die Zustimmung der Freien Wähler aus Bayern. Schließlich trat er am 31. Mai 2010 aufgrund der Kritik an seinen missverständlichen Äußerungen zu Bundeswehreinsätzen überraschend zurück.

\section{Köhlers Auftreten in der Öffentlichkeit}

In seiner ersten Rede nach der Vereidigung vor den Abgeordneten von Bundestag und Bundesrat ließ Köhler bereits ein ,aktives Amtsverständnis“ erkennen. So erklärte er, dass Deutschland dringend modernisiert werden müsse und es seine Pflicht als Bundespräsident sei, zu diesem Prozess beizutragen. Er hob hervor, dass das Reformprogramm der rot-grünen Koalition, die Agenda 2010, die 2003 eingeführt worden war, in die richtige Richtung weise und er es für nötig erachte, bei den erforderlichen Reformen Kurs zu halten. ${ }^{28}$ Dabei wird deutlich, dass Köhler von Anfang an von den politischen Möglichkeiten, die ihm sein Amt boten, regen Gebrauch zu machen suchte. So bemühte er sich bald, seiner Präsidentschaft deutliche Konturen zu geben und schreckte nicht davor zurück, (häufig) zu aktuellen tagespolitischen Themen Stellung zu nehmen. Im September 2004 sorgte Köhler für Schlagzeilen, als er in einem Interview sagte, er glaube die Unterschiede zwischen Ost- und Westdeutschland würden noch lange anhalten. Als nur kurze Zeit später bekannt wurde, dass Schröder darüber nachdachte, den 3. Oktober als Tag der deutschen Einheit aus wirtschaftlichen Gründen als Feiertag abzuschaffen und die Feierlichkeiten stattdessen am ersten

27 Vgl. Schuler, K.: Ein äußerst knapper Sieg und viel Heiterkeit, http://www.zeit.de/. 28 Vgl. Köhler, H.: Wir können in Deutschland vieles möglich machen, 01.06.2004. 
Sonntag im Oktober stattfinden zu lassen, mischte sich Köhler wieder in die Debatte ein und sprach sich entschieden gegen diese Überlegungen aus. Ende November kritisierte der Bundespräsident den Vorschlag des nordrheinwestfälischen Ministerpräsidenten Jürgen Rüttgers, den Bezug von Arbeitslosengeld von der Zahl der Beitragsjahre abhängig zu machen, und wies darauf hin, dass die Arbeitslosenversicherung kein individueller Sparvertrag sei. ${ }^{29} \mathrm{Im}$ Juni 2007 schlug Köhler sogar eine Verfassungsänderung in Bezug auf das Bundespräsidentenamt vor, indem er sich für direkte Wahlen des Staatsoberhauptes aussprach. ${ }^{30}$ In Anbetracht der deutschen Geschichte bzw. der „Weimarer Erfahrungen" war es kaum überraschend, dass dieser Vorschlag nicht nur beim Großteil der politischen Elite, sondern auch bei Staatsrechtlern ein wenig positives Echo fand. ${ }^{31}$ Nur ein paar Wochen später übte Köhler explizit Kritik an Bundesinnenminister Wolfgang Schäuble, indem er diesen mangelnde Sensibilität bei den Gesprächen über das Anti-Terror-Gesetz vorwarf. ${ }^{32}$ Im November 2007 kritisierte er die seiner Meinung nach unvernünftig hohen Managergehälter; im Dezember des gleichen Jahres warnte er vor den Risiken der Einführung des Mindestlohns. In seiner Berliner Rede im Juni 2008 lobte Köhler erneut die umstrittene Agenda 2010. Zugleich forderte er ,die weitere Modernisierung Deutschlands“ und eine Agenda $2020 .^{33}$ Als das Ausmaß der Finanzkrise allmählich bekannt wurde, verurteilte er (als früherer Geschäftsführender Direktor des Internationalen Währungsfonds) die Finanzmärkte, die sich seiner Meinung nach zu einem „Monster“ entwickelt hätten. Zugleich forderte er radikale Reformen von den Banken. Seine Berliner Rede im März 2009 nahm er dann zum Anlass, die politischen Parteien davor zu warnen, die Wirtschaftskrise als Kulisse für „Schaukämpfe“" zu verwenden. Zudem forderte er nicht nur eine verstärkte Regulierung des Marktes, sondern auch „Regeln und Moral“ ${ }^{34}$

Nachdem Köhler zudem den Bundestag vorzeitig auflöste und sich wiederholt weigerte, von der Großen Koalition verabschiedete Gesetze auszufertigen, entstand der Eindruck, dass das Amt des Bundespräsidenten unter seiner Amtsfüh-

29 Vgl. Köhler, H.: Ansprache bei der Vollversammlung des Deutschen Industrie- und Handelskammertages, 22.11.2006.

30 Vgl. ARD: Sabine Christiansen, 24.06.2007.

31 Unter den Spitzenpolitikern sprachen sich nur Guido Westerwelle von der FDP und Oskar Lafontaine von der Linkspartei für die Idee aus.

32 Vgl. Köhler, H.: ZDF-Sommerinterview - Der Bundespräsident im Gespräch mit Paul Hahne, 15.07.2007.

33 Vgl. Köhler, H.: Arbeit, Bildung, Integration, 17.06.2008.

34 Vgl. Köhler, H.: Die Glaubwürdigkeit der Freiheit, 24.03.2009. 
rung erheblich politisiert wurde. In der Folge wurde Köhler als der ,politischste Präsident, den das Land je hatte“35 oder sogar als Teil einer neuen ,,außerparlamentarischen Opposition“ "etikettiert. ${ }^{36}$ Allerdings waren seine Entscheidung, den Bundestag aufzulösen, und - wenn auch in geringerem Maße - seine Weigerung, zwei Gesetze auszufertigen, keineswegs so politisch wie die Medienreaktion dies vermuten ließ.

Nach seiner Wiederwahl zum Bundespräsidenten wurde Köhler deutlich ruhiger und unauffälliger. Zwar äußerte er sich zu verschiedenen Themen, kritisierte etwa die schwarz-gelbe Bundesregierung, die Finanzbranche sowie die Umsetzung des Bologna-Prozesses und plädierte für stärkere Kontrollen der Finanzmärkte, mehr Haushaltsdisziplin, (vor dem Hintergrund des Amoklaufs in Winnenden) eine „Kultur der Achtsamkeit“ und eine notwendige Reformpolitik. Letztlich aber verpufften die wenigen, nicht energisch genug vorgetragenen Äußerungen ohne größere Reaktion. Während sich Köhler in seiner ersten Amtszeit häufig in Details der Alltagspolitik einmischte, versäumte er es in seiner zweiten Amtszeit, zu zentralen, übergreifenden Entwicklungen und Problemen lautstark und vehement Stellung zu beziehen. Seit die schwarz-gelbe Bundesregierung im Amt war, insbesondere in den letzten Monaten vor seinem Rücktritt, war der Bundespräsident so unauffällig, dass er von der Opposition offen dafür kritisiert wurde und die BILD-Zeitung fragte „Wo ist eigentlich Super-Horst?“ ${ }^{37}$. Die bei der Ernennung des neuen Bundesverfassungsgerichtspräsidenten Andreas Voßkuhle erfolgte Ankündigung, in der zweiten Amtszeit den Schwerpunkt auf die Erhaltung und Verbesserung der Vitalität unserer Demokratie zu setzen ${ }^{38}$, wirkte in Zeiten der Wirtschafts- und Finanzkrise sowie angesichts der mangelnden Handlungsfähigkeit der schwarz-gelben Bundesregierung wie eine „Themenverfehlung“. Zusammenfassend ist festzuhalten, dass es dem Bundespräsidenten in seiner zweiten Amtszeit kaum mehr gelang, wesentliche Akzente zu setzen.

35 Fischer, S.: Bundespräsident Köhler: Zupacken, Angreifen, Dazwischengrätschen, http://www.spiegel. $\mathrm{de} /, 14.12 .2006$.

36 Vgl. Geis, M./Ulrich, B.: Die neue Apo, http://www.zeit.de/, 21.01.2007

37 Bild-Zeitung, zit. in: Bannas, G.: Verstrickt in Amt und Würden, in: FAZ, 01.06.2010, 3.

38 Vgl. „Köhler nennt neuen Schwerpunkt“, http://www.zeit.de/. 


\section{Köhlers Entscheidung, den Bundestag aufzulösen}

Da das Grundgesetz eine Selbstauflösung des Bundestages vor Ende einer Legislaturperiode nicht vorsieht, kann, wie aufgezeigt, eine vorzeitige Auflösung nur vom Bundespräsidenten veranlasst werden, wenn der Bundeskanzler ihm dies nach einer gescheiterten Vertrauensfrage vorschlägt (Art. 68 Abs. 1 GG) - oder nachdem auf der dritten Stufe des Kanzlerwahlverfahrens kein Bundeskanzler mit mehr als der Hälfte der Stimmen gewählt werden konnte (Art. 63 Abs. 4 GG). Nach dem katastrophalen Wahlergebnis der SPD bei der Landtagswahl in Nordrhein-Westfalen am 22. Mai 2005 gab Gerhard Schröder nach Konsultation mit dem SPD-Parteivorsitzenden Franz Müntefering bekannt, dass er beabsichtige, die Vertrauensfrage zu stellen, um - nach ihrem intendierten Scheitern Neuwahlen herbeizuführen. Die Entscheidung, auf diesem Weg eine Auflösung des Bundestages anzustreben, war nicht nur wegen der politischen Mittel (der Instrumentalisierung der Vertrauensfrage), sondern auch wegen der potentiellen politischen Folgen dieser Strategie umstritten. Vor allem Außenminister Joschka Fischer war über die Monate der Ungewissheit, die der Vertrauensfrage folgen könnten, besorgt. Schröder selbst bezeichnete die Entscheidung als eine der schwersten seiner gesamten politischen Laufbahn, hielt sie aber für die einzige Möglichkeit, seinen Rücktritt wegen mangelnder Unterstützung in den eigenen Reihen zu vermeiden. ${ }^{39}$ In seiner Rede, in der er die Gründe für das Stellen der Verfrauensfrage darlegte, machte Schröder klar, dass sowohl die öffentliche Unterstützung für seine Regierungskoalition als auch die Unterstützung in den eigenen Reihen seit der Einführung der Agenda 2010 stark nachgelassen habe. Er argumentierte, dass das Wahlergebnis vom 22. Mai die Möglichkeit, seine Politik erfolgreich fortzusetzen, ernsthaft in Zweifel gefährdete, vor allem aufgrund der sehr knappen Mehrheit seiner Koalition im Bundestag und der ständigen Drohungen von Mitgliedern seiner eigenen Partei, sich bei Abstimmungen der Stimme zu enthalten, oder gegen die Regierungspolitik zu votieren. Eine vorgezogene Wahl würde es daher dem Volk ermöglichen, die Richtung der zukünftigen Regierungspolitik bestimmen zu können. Um vorzeitige Wahlen zu erreichen, entschied sich Schröder für die Vertrauensfrage - und damit gegen den Rücktritt, der ebenfalls (im Falle einer gescheiterten Neuwahl eines Bundeskanzlers mit absoluter Mehrheit) zu Neuwahlen hätte führen können. Schröder bezeichnete diesen Weg jedoch als ,der Würde des Hohen Hauses nicht angemes-

39 Vgl. Schröder, G.: Entscheidungen. Mein Leben in der Politik, Hamburg, 2006, 374. 
sen“40. Bei der „konstruierten“ Vertrauensfrage, die am 1. Juli 2005 gestellt wurde, versagte der Bundestag Schröder - wie intendiert - das Vertrauen. 296 Bundestagsabgeordnete unterstützten die Vertrauensfrage nicht, 151 stimmten dafür und 296 Abgeordnete enthielten sich auf Anweisung des SPD-Parteivorsitzenden Müntefering ihrer Stimme.

Der dann über die Auflösung des Bundestages zu befindende Bundespräsident nutzte die vollen drei Wochen, die ihm zur Verfügung standen, um eine Entscheidung zu treffen. Am 21. Juli 2005 gab Köhler seinen Entschluss bekannt, dem Wunsch des Kanzlers nachzukommen, den Bundestag aufzulösen und vorgezogene Wahlen stattfinden zu lassen. In seiner Erklärung wies er allerdings darauf hin, dass eine Niederlage des Bundeskanzlers bei der Vertrauensabstimmung für eine Auflösung des Bundestages allein nicht ausreiche. Unter Berufung auf die Entscheidung des Bundesverfassungsgerichts von 1983 argumentierte er, dass die politischen Kräfteverhältnisse im Bundestag die Handlungsfähigkeit des Kanzlers so beeinträchtigen oder sogar lähmen müssten, dass ,er eine von stetiger Zustimmung der Mehrheit getragene Politik nicht sinnvoll verfolgen kann“. Schließlich fügte er hinzu, dass genau dies die Lage sei, in der sich Bundeskanzler Schröder sähe. In diesem Zusammenhang machte er auch deutlich, dass der Bundespräsident laut dem Bundesverfassungsgerichtsurteil von 1983 die Beurteilung des Kanzlers berücksichtigen müsse, wenn keine andere Einschätzung eindeutig vorzuziehen sei. Ferner machte er deutlich, dass mit einer Neuwahl „dem Wohl unseres Volkes“ am besten gedient sei. ${ }^{41}$ Somit war aus der Sicht des Bundespräsidenten eine Bundestagsauflösung nicht nur verfassungsrechtlich möglich, sondern auch politisch sinnvoll. Folglich löste Köhler - obwohl er dies nicht hätte tun müssen - den Bundestag auf. Die Entscheidung des Bundespräsidenten bzw. dessen Einschätzung der verfassungsrechtlichen Voraussetzungen für die Auflösung des Bundestags wurde durch das nachfolgende Urteil des Bundesverfassungsgerichtes vom 25. August 2005 bestätigt, das den Einspruch zweier Bundestagsabgeordneter gegen die Bundestagsauflösung zurückwies. Dabei stellte das Bundesverfassungsgericht klar, dass das Grundgesetz einen Kanzler an der Spitze der Regierung vorsehe, der in der Lage sei, effektiv zu regieren, da er sich auf eine Mehrheit im Bundestag verlassen könne. Ob Letzteres der Fall sei, könne „,von außen nur teilweise beurteilt werden“. Jedenfalls sei es - gemessen am Sinn des Art. 68 GG - ,nicht zweckwidrig“, wenn ein Kanzler

40 Zit. nach Schröder, G., in: Deutscher Bundestag: Plenarprotokoll 15/185, 17466.

41 Vgl. Köhler, H.: Auflösung des 15. Deutschen Bundestages, 21.07.2005. 
in Erwartung von Niederlagen bei künftigen Abstimmungen die Vertrauensfrage stelle. $^{42}$

Vor dem Hintergrund der Urteile des Bundesverfassungsgerichts von 1983 und 2005, die die materiellen Bedingungen für eine Bundestagsauflösung nach einer gescheiterten Vertrauensfrage festlegen (die Existenz einer politischen Instabilität), war Köhler eindeutig berechtigt, den Bundestag aufzulösen. Bedenkt man zudem, dass mehrheitlich alle im Bundestag vertretenen Parteien und eine breite Mehrheit der Bevölkerung ${ }^{43}$ für eine vorgezogene Wahl plädierten, wird deutlich, dass Köhlers Entscheidung, der Bitte des Kanzlers nachzukommen, nicht nur legal, sondern auch politisch gut vertretbar war. Jedenfalls war sie deutlich nachvollziehbarer und weniger "politisch“ als dies manche Bewertungen in den Medien glauben machen wollten. Letztlich wäre die Entscheidung des Bundespräsidenten, die Bitte des Kanzlers abzulehnen, um ein Vielfaches ,politischer“ gewesen.

\section{Köhlers Weigerung, Gesetze auszufertigen}

Gegen Ende 2006 kam Bundespräsident Köhler aufgrund seiner wiederholten Weigerung Gesetze auszufertigen, die die Große Koalition verabschiedet hatte, erneut in die Schlagzeilen. Ihm wurde vorgeworfen, das Amt des Bundespräsidenten zu politisieren. Im Januar 2005 hatte Köhler ein Gesetz zur Luftsicherheit zwar ausgefertigt, gleichzeitig jedoch eine Prüfung durch das Bundesverfassungsgericht empfohlen (das Gesetz wurde anschließend vom Bundesverfassungsgericht als verfassungswidrig erklärt). ${ }^{44}$ In zwei weiteren Fällen weigerte er sich jedoch, das vorliegende Gesetz auszufertigen.

Am 24. Oktober 2006 weigerte sich Köhler, das Gesetz zur Privatisierung der Flugsicherung in Deutschland auszufertigen, das am 7. April im Bundestag verabschiedet worden war. Dabei wies er darauf hin, dass das Gesetz seiner Meinung nach evident verfassungswidrig sei. Köhler identifizierte einen materiellen Verstoß gegen Art 87 Abs. 1 GG, der vorsieht, dass die Zuständigkeit für die

42 Vgl. Bundesverfassungsgerichtsentscheidung: $2 \mathrm{BvE} \mathrm{4/05,} 2 \mathrm{BvE} 7 / 05$ vom 25.08.2005, http://www. bverfg.de/.

43 Auf die Frage „Halten Sie eine Neuwahl des Bundestages in der jetzigen politischen Situation für dringend geboten?" antworteten 65\% der Befragten mit 'ja' und nur 31\% mit 'nein' (TNS Infratest für den Spiegel vom 28. bis 30. Juni), vgl. Palmer, H.: Plan B, in: Der Spiegel, 59/27 (2005), 32-33, hier 33.

44 Vgl. Bundesverfassungsgerichtsentscheidung: 1 BvR 357/05 vom 15. Februar 2006, http://www. bverfg.de/; sowie Bundespräsidialamt: Bundespräsident Horst Köhler unterzeichnet Luftsicherheitsgesetz, 12.01.2005. 
Flugsicherung beim Bund liegt. Köhlers Weigerung, das Gesetz auszufertigen, konnte kaum überraschen. Schon im Rahmen der Debatte im Bundestag wurden Zweifel an der Verfassungsmäßigkeit des Gesetzes laut. Nachdem er bereits im Vorfeld die Verfassungsmäßigkeit des Gesetzes angezweifelt und um weitere Erklärungen gebeten hatte ${ }^{45}$, weigerte er sich, das Gesetz auszufertigen. Es war das erste Mal seit 1991, dass ein Bundespräsident von diesem Recht Gebrauch machte.

Am 8. Dezember 2006 weigerte sich Köhler, ein Gesetz auszufertigen, das als Folge des „Gammelfleischskandals“ zustande kam und zum Ziel hatte, das Informationsrecht der Verbraucher zu stärken. ${ }^{46}$ Köhler argumentierte, dass das Gesetz materiell gegen Art. 84 GG verstoße, der im Rahmen der Föderalismusreform der Großen Koalition erst im September 2006 in Kraft getreten war. ${ }^{47}$ Schließlich sah das Gesetz vor, dass den Kommunen Aufgaben vom Bund übertragen werden, was aufgrund der seit der Föderalismusreform geänderten Verfassungslage nicht mehr möglich war. Mit anderen Worten: Die Regierung hatte die Konsequenzen ihrer eigenen Reform nicht bedacht. Köhler war bei weitem nicht der einzige und auch nicht der erste, der Bedenken äußerte. Bereits zuvor hatten die Bundesratsmitglieder Berlins, Sachsen-Anhalts und Schleswig-Holsteins ihre Bedenken bezüglich der Verfassungsmäßigkeit des neuen Gesetzes zum Ausdruck gebracht. Gleichwohl wurde das Gesetz am 22. September 2006 vom Bundesrat verabschiedet.

Mitglieder der Regierungsparteien drückten ihren Respekt vor der Entscheidung des Bundespräsidenten aus, zeigten sich aber über sein Eingreifen empört. Besonders interessant ist dabei die Tatsache, dass sich die Reaktionen weniger auf eine Verteidigung des Gesetzes richteten, sondern eher auf die Frage, ob Köhler mit seiner Weigerung, das Gesetz auszufertigen, seine Kompetenzen überschritten habe. So wies etwa Peter Struck, Vorsitzender der SPD-Bundestagsfraktion, darauf hin, dass das Bundesverfassungsgericht in Karlsruhe und „niemand sonst“ über die Verfassungsmäßigkeit von Gesetzen zu entscheiden habe. ${ }^{48}$

45 Vgl. Bannas, G.: Bundespräsident Köhler. Nicht die erste Rüge, auf: http://www.faz.net/.

46 Vgl. Bundespräsidialamt: Bundespräsident Horst Köhler fertigt Gesetz zur Neuregelung des Rechts der Verbraucherinformation nicht aus, 08.12.2006.

47 Ebd.

48 Vgl. „Amtsverständnis des Bundespräsidenten: Verärgerung in der Koalition über Köhler“, http://welt .de/, 13.12.2006. 
Materiell erschien es in beiden Fällen gerechtfertigt, dass sich der Bundespräsident weigerte, die Gesetze auszufertigen. Beide wiesen erhebliche, möglicherweise auf die große Mehrheit im Bundestag zurückzuführende verfassungsrechtliche Mängel auf. Wenn die Regierungskoalition über eine geringere Mehrheit verfügt hätte, wäre sie vermutlich vorsichtiger vorgegangen. Letztlich hatte Köhler gute Gründe, seine Unterschrift zu verweigern, wenngleich dies nicht zwingend nötig war. Er hätte auch dem Beispiel von Johannes Rau folgen können, der 2002 das Zuwanderungsgesetz unterzeichnete, obwohl man sein Zustandekommen - wie es später auch das Bundesverfassungsgericht tat - ohne Frage als „Zweifelsfrei und offenkundig“ verfassungswidrig hätte einordnen können.

Die Art und Weise, mit der Köhler von seiner Prüfungskompetenz bei der Ausfertigung von Gesetzen Gebrauch machte, erweckte schließlich den Anschein, er sei ein politischeres Staatsoberhaupt als seine Vorgänger und wirkte letztlich weit über die beiden Entscheidungen hinaus. So wurde die „rote Köhler-Karte“ in der Folge zusehends in den Ausschüssen des Bundestages gezogen, ${ }^{49}$ mit dem Resultat, dass die Große Koalition sehr viel umsichtiger wurde, so etwa im Rahmen der Diskussionen um die Verfassungsmäßigkeit des Gesetzes zur Finanzierung von Unterkunftskosten für Hartz IV-Empfänger deutlich wurde.

Horst Köhler ließ auch in anderen Fällen erkennen, dass er - wie er selbst sagte kein „Unterschriftenautomat“ sei. ${ }^{50}$ So hatte er das Gesetz zur Erschwerung des Zugangs zu kinderpornografischen Inhalten in Kommunikationsnetzen (Zugangserschwerungsgesetz), das noch von der Großen Koalition verabschiedet worden war, zunächst nicht unterzeichnet und um ergänzende Informationen gebeten. Als die in der Zwischenzeit neu gebildete schwarz-gelbe Bundesregierung mitteilte, ohnehin eine andere Lösung anzustreben und die Internetsperren auszusetzen, ${ }^{51}$ fertigte Köhler das Gesetz aus, da er „,keine durchgreifenden verfassungsrechtlichen Bedenken “52 feststellen konnte. Dabei ist das Verfahren, ein bestehendes Gesetz nicht zur Anwendung zu bringen, verfassungsrechtlich ebenso problematisch wie ein angreifbares Gesetz per se. ${ }^{53}$ Köhler hätte das Gesetz also mit gutem Grund zurückweisen können. Bei dem Zustimmungsgesetz zum Lissabon-Vertrag wartete der Bundespräsident eine Entscheidung des Bundes-

49 Vgl. Geis, M./Ulrich, B., a.a.O.

50 Vgl. Müller, R.: Kein Ersatzkaiser, kein Unterschriftenautomat, in: FAZ, 01.06.2010, 2.

51 Vgl. ,Schwarz-Gelb entsagt den Internetsperren“, http://www.zeit.de/.

52 Vgl. „Köhler unterzeichnet Gesetz zur Internetsperre“, http://www.zeit.de/.

53 Vgl. Müller-Neuhof, J.: Köhler verweigert seine Unterschrift, http://www.zeit.de/. 
verfassungsgerichts ab, das von verschiedenen Seiten, u.a. durch Peter Gauweiler, Gregor Gysi und Oskar Lafontaine, angerufen worden war. Das Bundesverfassungsgericht erklärte den Lissabon-Vertrag für verfassungskonform, verlangte jedoch eine Überarbeitung der deutschen Begleitgesetze, in denen die Mitbestimmung von Bundestag und Bundesrat bei der Umsetzung europäischer Rechtsakte in Deutschland geregelt ist. Dem positiven Votum aus Karlsruhe und einer entsprechenden Überarbeitung der Begleitgesetze folgte schließlich auch die Unterschrift des Bundespräsidenten.

\section{Köhlers Entscheidung, frühere RAF-Terroristen nicht zu begnadigen}

Nachdem Köhler wegen seiner Entscheidung, den Bundestag aufzulösen, und seiner wiederholten Weigerung, von der Großen Koalition verabschiedete Gesetze auszufertigen, die Aufmerksamkeit der Medien bereits verstärkt auf sich gezogen hatte, fanden die Diskussionen über eine mögliche Begnadigung von früheren RAF-Terroristen, insbesondere eine mögliche Begnadigung von Christian Klar, im Vorfeld der Entscheidung des Bundespräsidenten nicht nur in parteipolitischen Debatten, sondern auch in der Öffentlichkeit großes Interesse. Klar war als Mitglied der RAF an den politisch motivierten Morden an Hanns-Martin Schleyer, Siegfried Buback und Jürgen Ponto, alle in den siebziger Jahren Repräsentanten der Bonner Republik, beteiligt. Wie bereits erwähnt, ist die Befugnis einzelne Täter zu begnadigen, wie es Art. 60 Abs. 2 GG vorsieht, keine politische Befugnis im engeren Sinn, sondern eine rechtliche Befugnis mit potentiell erheblichen politischen Auswirkungen. Obwohl Reue nicht als notwendige Vorbedingung für die Begnadigung durch den Bundespräsidenten gilt, spielte sie trotzdem eine wichtige Rolle bei dessen Meinungs- und Willensbildungsprozess - und in der öffentlichen Debatte. CSU-Generalsekretär Markus Söder demonstrierte die politische Dimension der Entscheidung, indem er sogar so weit ging, Druck auf Köhler auszuüben. Schließlich deutete er an, dass eine positive Entscheidung für Christian Klar Köhlers Chancen auf eine Wiederwahl negativ beeinflussen könnte. Ob sich der Bundespräsident bei seiner Entscheidung tatsächlich beeinflussen ließ, ist nicht festzustellen. Jedenfalls gab Köhler kurz nach seinem Treffen mit Klar am 7. Mai 2007 seine Entscheidung bekannt, Christian Klar - wie auch Birgit Hogefeld, die später ein Gnadengesuch an den Bundespräsidenten richtete - nicht zu begnadigen. ${ }^{54}$

54 Vgl. Bundespräsidialamt: Bundespräsident Horst Köhler hat über die Gnadengesuche von Herrn Christian Klar und von Frau Birgit Hogefeld entschieden. 


\section{Horst Köhlers Rücktritt}

Am 31. Mai 2010 trat Horst Köhler mit sofortiger Wirkung von seinem Amt zurück. Nach einem Kurzbesuch im Bundeswehrfeldlager in Masar-i-Scharif in Afghanistan hatte er in einem Interview gesagt, dass ,im Zweifel im Notfall auch militärischer Einsatz notwendig ist, um unsere Interessen zu wahren, zum Beispiel freie Handelswege [...]. ${ }^{\text {(55 }}$ Daraufhin wurde er mit einiger zeitlicher Verzögerung in ungewöhnlich (aber nicht erkennbar übergebührlich) scharfer Form von Politikern und Journalisten kritisiert. Nur wenige Politiker der Regierungskoalition (etwa Guido Westerwelle) nahmen ihn in Schutz, viele (auch die Bundeskanzlerin) schwiegen oder nannten die Äußerungen missverständlich, während einige Vertreter aus dem Oppositionslager und Journalisten - mehr oder weniger deutlich - darauf hinwiesen, dass Köhler zu Unrecht verfassungswidrige Wirtschaftskriege befürworte und seine Äußerungen zurücknehmen sollte. Dabei wurden auch Vergleiche zu Heinrich Lübke gezogen, der in seiner zweiten Amtszeit - erkennbar krankheitsbedingt - nicht selten durch fragwürdige Äußerungen auffiel. Nach einem (unglücklichen) Versuch, die Äußerungen nachträglich auf den Einsatz der Bundeswehr im Rahmen der Operation Atalanta gegen Piraten am Horn von Afrika zu beziehen, ${ }^{56}$ tat Köhler jedoch, was letztlich niemand forderte oder erwartete und vor ihm kein Bundespräsident tat: ${ }^{57} \mathrm{Er}$ trat mit sofortiger Wirkung zurück - allerdings nicht wegen seiner missverständlichen Äußerungen, sondern aufgrund der sich hierauf richtenden Kritik: „Die Kritik geht aber so weit, mir zu unterstellen, ich befürwortete Einsätze der Bundeswehr, die vom Grundgesetz nicht gedeckt wären. Diese Kritik entbehrt jeder Rechtfertigung. Sie lässt den notwendigen Respekt für mein Amt vermissen. “58 Selbst Gregor Gysi hielt den Rücktritt für „etwas übertrieben“. ${ }^{59}$ Es ist unwahrscheinlich, dass der offizielle auch der tatsächliche Grund für den Rücktritt war. Viele Faktoren mögen dazu beigetragen haben: Friktionen mit bzw. mangelnder Rückhalt seitens der schwarz-gelben Bundesregierung, Personal- und Führungsprobleme im Bundespräsidialamt, eine tiefe Enttäuschung über die geringen Einflussmöglichkeiten sowie eine gewisse Amtsmüdigkeit, die möglicherweise auch

55 Köhler, H., zit. in: „Bundespräsident Köhler zurückgetreten“, in: FAZ, 01.06.2010, 1.

56 Vgl. „Militäreinsätze für deutsche Wirtschaftsinteressen?“, http://www.zeit.de/.

57 Heinrich Lübke erklärte - gesundheitlich stark angeschlagen und kurz vor dem Ende seiner vollen zweiten Amtszeit - am 14.10.1968 seinen Rücktritt zum 30.06.1969. Vgl. „Staatsorgane in Zugzwang“, in: FAZ, 01.06.2010, 2.

58 Vgl. „Die Erklärung von Bundespräsident Köhler im Wortlaut“, in: FAZ, 01.06.2010, 2.

59 Vgl. „Präsident nicht sakrosankt gegenüber Kritik“, in: FAZ, 01.06.2010, 2. 
Köhlers Zurückhaltung während der zweiten Amtszeit begründete. Die Kritik am Rücktritt Köhlers war in den Medien dann zum Teil weitaus heftiger als die Kritik an seinen missverständlichen Äußerungen. ${ }^{60}$

\section{Schluss}

Der Bundespräsident der Bonner/Berliner Republik ist nicht so mächtig wie der Reichspräsident der Weimarer Republik, er ist aber bei weitem nicht ohnmächtig. Dies lässt sich an der Vielfalt von Kompetenzen dokumentieren, von denen Horst Köhler während seiner ersten Amtszeit Gebrauch machte.

Heute sollte kein Zweifel daran bestehen, dass das Amt des Bundespräsidenten seit Köhlers Wahl im Jahr 2004 eine gewisse Politisierung erfuhr. Dies dürfte allerdings mehr auf seine Kommentare zur Tagespolitik denn auf die Ausnutzung seiner formalen Kompetenzen zurückzuführen sein. Die Entscheidung des Bundespräsidenten, den Bundestag aufzulösen, um vorzeitige Wahlen zu ermöglichen, war mit dem Grundgesetz wie auch der Rechtsprechung des Bundesverfassungsgerichts konform und wurde von einem weitgehenden Konsens unter den politischen und wirtschaftlichen Eliten, aber auch seitens der Öffentlichkeit getragen. Diese Entscheidung ist mithin kein Indikator, dass Köhler im Vergleich zu seinen Vorgängern eine Ausweitung der Macht des Bundespräsidenten anstrebte. Im Gegenteil, eine Entscheidung gegen die Auflösung des Bundestages hätte das Amt deutlich stärker politisiert. Seine Weigerung, innerhalb von zwei Monaten zwei Bundesgesetze aufgrund von Zweifeln an ihrer materiellen Verfassungsmäßigkeit auszufertigen, zeigt dagegen mehr politischen Interventionsgeist. Die Befugnis des Bundespräsidenten, die Verfassungsmäßigkeit von Gesetzen zu überprüfen und diese gegebenenfalls nicht auszufertigen, ist allerdings verfassungsrechtlich verankert, wird in der Staatsrechtslehre breit bejaht und wurde bereits von Köhlers Vorgängern genutzt. Zudem konnte der Bundespräsident die Nicht-Ausfertigung in beiden Fällen stichhaltig begründen.

Gleichwohl hat Köhler in der Gesamtschau das Amt des Staatsoberhauptes politisiert - vor allem durch seine Einmischung in die Tagespolitik während seiner ersten Amtszeit. Die Art, wie Köhler das Amt des Bundespräsidenten ausfüllte, wird allerdings nicht so sehr die Erinnerung an seine Präsidentschaft prägen als die Form, in der von diesem Amt zurücktrat.

60 So wurde er in der FAZ als ,präsidialer Rohrkrepierer“ bezeichnet. Vgl. Hanfeld, M.: Bundespräsident Köhler zurückgetreten, in: FAZ, 01.06.2010, 33. 